documentation of the discussions had with patients and carers about the risks and benefits of using antipsychotic medications for management of BPSD. A teaching session was held at the team meeting to highlight the risks and benefits. The team will ensure that they provide a health board approved leaflet to each patient and carer following their discussion. Only 73\% of the patients had a CAIR form in their notes and the team favour the original version. The team will revert back to using the original version of the CAIR form as it has more space allocated to document ongoing reviews. We will re-audit in 6 months time.

\section{On-call medical seclusion reviews: are we meeting MHA code of practice (COP) requirements? \\ Oliver Turner ${ }^{1 \star}$ and David Leung ${ }^{2}$ \\ ${ }^{1}$ Leeds and York Partnership NHS Foundation Trust and ${ }^{2}$ PICU, Newsam Ward 1, Leeds and York Partnership NHS Foundation Trust ${ }^{*}$ Corresponding author.}

doi: 10.1192/bjo.2021.321

Aims. Are Junior Trainee, Medical Seclusion Reviews complaint with MHA COP Criteria?

Objectives. Are we seeing newly secluded patients on time?

Are we documenting these reviews in clinical notes?

Do documented reviews meet criteria stated by the MHA COP 26.133 ?

Are we informing Higher Trainees of the need for MDT reviews? Background. Seclusion is an important aspect of inpatient care. MHA COP Chapter 26 provides guidance for documenting seclusion reviews, ensuring safeguards are in place to protect patient's safety and human rights. Secluded patients require a medical review within 1 hour, and four hourly thereafter, until a higher trainee or Consultant undertake an MDT Review. In our Trust, LYPFT, trainees undertake these reviews. There is noted discrepancy in seclusion review documentation. This audit identifies our compliance with time limits, and whether documentation meets the required criteria in the MHA Code of Practice

Method. Our Sample includes all Out-of-Hour Junior Trainee Medical Seclusion Reviews between 01/01/20 and 01/04/20 at LYPFT. Seclusions were identified from on call logs, and clinical notes were reviewed for a documented seclusion review. The date and time of seclusion are recorded, whether a 1 or 4 hourly review, and the time of review. We recorded any mention of: physical health; mental state; observation levels; recent medication; medication side effects; risk to others; risk to self and the need for ongoing seclusion. Result. 56 episodes of seclusion were identified; all 56 had a documented medical seclusion review. 49 reviews were on time, 4 were late with a documented reason, and 3 were late without. There was documentation of the Higher Trainee being informed in 53 reviews.

No seclusion reviews mentioned all MHA COP criteria. We more frequently mentioned patients' physical health (51), psychiatric health (52) and need for seclusion (54). 46 seclusion reviews mentioned risk of harm to others; only 3 mentioned risk of self-harm. 25 seclusion reviews mentioned medication, and 5 mentioned review for side effects. 5 seclusion reviews mentioned observation levels.

Conclusion. Our Junior Doctor Seclusion Reviews were not meeting the MHA Code of Practice Criteria, and we believe this to largely be due to lack of awareness of the standards. As such, results have been disseminated to Junior trainees in weekly teaching. We created a medical seclusion review template, adopted by the Trust, to ensure documentation compliance with the MHA COP. Junior doctor inductions now include a presentation regarding Seclusion, the reviews and documentation. We will re-audit in 12 months.
Early intervention in psychosis team (EIT): pathways to care

Chloe Uffendell ${ }^{\star}$ and John Stevens

MerseyCare NHS Trust

${ }^{\star}$ Corresponding author.

doi: 10.1192/bjo.2021.322

Aims. The main aim of this study was to investigate whether the EIT access and waiting time standard ( $>60 \%$ of people experiencing first episode psychosis (FEP) are treated with a NICE-approved care package within two weeks of referral) was being met within Liverpool EIT.

We also wanted to understand the pathway to treatment within EIT services, identify delays in the process of triage/assessment/ $\mathrm{MDT} / \mathrm{medical}$ review and implement changes to reduce delays.

Method. This study was a retrospective cross-sectional audit of all patients accepted on to the FEP pathway following MDT discussion in the Liverpool EIT Teams across May and June 2020.

Case notes were analysed for delays in referral, engagement with assessment and care-coordinators, as well as prescriber review offering medication. The data were collated and analysed before implementing changes.

Result. 40 patients presented as FEP in May and June 2020, 6 were excluded due to an extended inpatient stay.

Within the remaining patient cohort $(n=34), 64.7 \%$ of patients were engaged with a care package within 14 days. Only $14.7 \%$ of patients received an offer of medication within 14 days, the mean time to be offered medication was 39 days.

$26 \%$ of patients first contact within MerseyCare Trust was with EIT, $74 \%$ presented elsewhere. $24 \%$ instead presented to liaison psychiatry from A\&E departments, $18 \%$ to the single point of access team, 9\% to criminal justice liaison team (CJLT) and 9\% to North West Ambulance Service triage car.

$29 \%$ of referrals came from the community (GP and counselling services), $15 \%$ from CRHT (crisis resolution and home treatment team), $14 \%$ from CJLT, $12 \%$ from urgent care team, $9 \%$ from liaison psychiatry.

Conclusion. The Access and Waiting time standard was met. However, this study showed that patients were not being referred to EIT at first point of contact. This study shows $26 \%$ of service users first presented to liaison psychiatry, yet only $1 / 3$ of those were immediately referred to EIT, the remainder being later referred by other services e.g. CRHT.

In addition to referral delays, lack of medical practitioner availability caused significant delays in arranging medical reviews, delaying patients access to medication.

The changes implemented to address these issues included educating MerseyCare services in the early recognition of psychosis to increase early referral. Non-medical prescribers' roles were developed to perform initial medical reviews in addition to doctors, allowing patients earlier medication access. This allowed 'urgent slots' to be developed, time set aside for emergencies enabling prompt review of urgent cases.

An audit of lithium prescribing practices in an old age psychiatry service highlighting renal impairment in this cohort

Leia Valentine*, John Cannon, Siobhan Marmion, Michelle Corcoran, Marguerite Cryan, Geraldine McCarthy and Catherine Dolan

Liscarney House, Psychiatry of Old Age Service

${ }^{\star}$ Corresponding author.

doi: 10.1192/bjo.2021.323 